\title{
ARRAY JOINT DETECTION FOR C/TDMA SYSTEMS IN UMTS ENVIRONMENTS
}

\author{
Carles Antón-Haro \\ José A.R. Fonollosa \\ Javier R. Fonollosa \\ Dept. of Signal Theory and Communications - Universitat Politècnica de Catalunya.* \\ c/ Gran Capità s/n. Campus Nord UPC. 08034 Barcelona (SPATN). \\ e-mail: \{carles,adrian,fono\}@gps.tsc.upc.es
}

\begin{abstract}
In this paper, two array-based schemes for intracell and intercell interference suppression are proposed. In both cases, the spatial and temporal characteristics of the propagation are jointly exploited by placing a narrowband beamformer prior to the corresponding data detection stage. In the first approach, Filtered Training Sequence Joint Detection Receiver (FTS-JDR), the beamformer is devoted to exclusively cancel out intercell interference. This way, intracell users can be jointly detected in a MMSE detection block. In con trast, the second algorithm, known as Filtered Training Sequence Multisensor Receiver (FTS-MR), aims to attenuate all the interferers in the beamforming stage which allows the user of interest to be detected following a MLSE strategy. In order to assess performance of the proposed schemes, a set of link-level computer simulations adopting FRAMES' proposal for UMTS air-interface as well as realistic channel models for third generation communication systems is provided. Simulation results indicate that lower BERs can be obtained by concentrating interference cancellation tasks in the beamforming block.
\end{abstract}

\section{INTRODUCTION}

A major challenge for wireless communications systems is the limited radio frequency spectrum to be shared by the users. This leads to the situation that, in practical systems, the capacity is mainly determined by the impact of the co-channel interference (CCI). In CDMA scenarios, CCI is a result of low cross-correlations among users spreading codes (Multiple Access Interference, MAI), whereas in TDMA systems co-channel interference is a result of frequency reuse.

In present days, the third generation mobile radio system (UMTS) undergoes standardization in Europe. The higher bit rate services (up to $2 \mathrm{Mb} / \mathrm{s}$ ) together with increased flexibility in defining customized services are the major dif-

*This work was partially supported by the European Commission under ACTS, Project: AC020 TSUNAMI (II), the National Plan of Spain, CICYT, TIC95-1022-C05-01, TIC96-0500-C1001, the Generalitat de Catalunya, CIRIT, 1996SGR-00096. The TSUNAMI (II) consortium is formed by the following organizations: ERA Technology, Motorola ECID, Orange PCS, Rober Bosch, France Telecom CNET, CASA, University of Bristol, Aalborg University, Universitat Politècnica de Catalunya and Wireless Systems International. ferentiating factors of third generation systems with respect to second generation[1][2].

Within the ACTS (Advanced Communications Technologies and Services) program, the FRAMES (Future RAdio wideband Multiple accEs System) project [3] have developed a proposal for the UMTS air interface. Two multiple access schemes, namely, Wideband TDMA with spreading features (WB-TDMA/CDMA, Mode 1) and Wideband CDMA (WB-CDMA, Mode 2) have been identified by FRAMES in order to meet UMTS requirements (c.f.[3]). In Mode 1, the basic transmission unit is one slot. This basic unit is then divided into smaller units, either subslots or by using spreading codes if the spreading feature is used (Fig.1). In Mode 2, the basic transmission unit in the resource space is code. In order to cope with different bit-rate requirements, parallel multicode is used on the downlink whereas, on the uplink, transmission resources are adaptively allocated by varying spreading ratio.

In the sequel, only Mode 1 with spreading feature will be taken into account. In this mode, a $4.615 \mathrm{~ms}$ frame is deployed. Depending on the environment and service, the frame and the burst can be dynamically adapted with link adaptation. There are two burst formats for the $1 / 8$ slot, one format for the $1 / 16$ slot and two burst formats for the $1 / 64$ slot. In the case of spreading, up to 8 (or even higher) spread bursts can be transmitted in one basic slot.

On the other hand, the TSUNAMI II (Technologies in Smart antennas for UNiversal Advanced Mobile Infrastructure - Part two) project is involved in the incorporation of adaptive antennas (AA) in third generation systems [4]. One of the benefits being important reductions in intracell/intercell interference which allows more users to be allocated within the same cell.

In this paper, some link-level simulation results for the Filtered Training Sequence Joint Detection Receiver (FTSJDR) and the Filtered Training Sequence Multisensor Receiver (FTS-MR) algorithms are presented. Both schemes have been applied to the uplink of a cellular system using FRAMES Mode 1 Spread speech burst-1 format. They employ standard vehicular (VEHx) and pedestrian (PEDx) channel models as specified by FRAMES, which have been modified to incorporate realistic angular description. The angular spread model has been derived in the channel sounding activities carried out within TSUNAMI (II). 


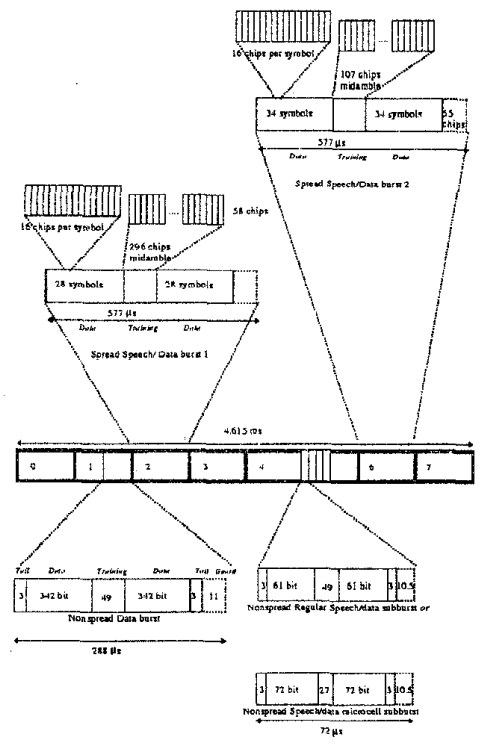

Figure 1. FRAMES proposal for UMTS air interface (Reproduced with permission of the FRAMES consortium.

\section{SIGNAL MODEL}

We consider a model for the general asynchronous multipleaccess channel being observed through a $P$ sensor array. The uplink signal received by sensor $p=1, \ldots, P$ is given by,

$$
r_{p}(t)=\sum_{n} \sum_{k=1}^{K} b_{k}[n] h_{k p}(t-n T, t)+\sigma w_{p}(t)
$$

where $h_{k p}(t)$ is the overall complex channel impulse response of user $k$ at sensor $p$, given by

$$
h_{k p}(t)=c_{k p}(t) * s_{k}
$$

that is, the convolution of its signature sequence $s_{k}$, with the physical channel and the transmitting and receiving filter impulse responses $c_{k p}(t)$. It incorporates the amplitude and the delay for user $k$ at sensor $p$, and its duration is assumed to be smaller or equal to $L$ symbol periods. The total number of active users is $K=K^{d}+K^{u}$ where $K^{d}$ is the number of desired users and $K^{u}$ is the number of undesired interferers. Transmitted data sequences are quaternary independent symbols $b_{k}[n] \in\{\pi / 4,3 \pi / 4,5 \pi / 4,7 \pi / 4\}$. The symbol rate is $1 / T$ and $w_{p}(t)$ is normalized temporal and spatially white Gaussian noise.

The multi-sensor multiple-access channel is sampled at the chip rate $f_{s}=1 / T_{c}=M / T$ to derive the $P \times 1$ snapshot $\mathbf{r}[n]=\left[r_{1}[n], \ldots, r_{P}[n]\right]^{T}$. A generic model for the angular characterisation of propagation channels is the Gaussian Wide-Sense Stationary uncorrelated scattering (GWSSUS) model. In this model, it is assumed that the radio wave frorn user $k$ propagates through a number of scatterers with gains $\tilde{\alpha}_{m}$, delays $l_{k, m} / c$ and azimuth angles $\theta_{m}$ (as seen from the base). The scatterers are grouped into $Q$ uncorrelated clusters, $\Omega_{q}$, with delay differences within each cluster that cannot be resolved for the selected transmission bandwidth (see [4]). In this situation, the multidimensional channel taps, $c_{k l}=\left[c_{k 1}\left(l T_{c}\right), \ldots, c_{k P}\left(l T_{c}\right)\right]^{T}$, can be expressed as

$$
\begin{aligned}
\mathbf{c}_{k l} & =\sum_{q=1}^{Q} \sum_{m \in \Omega_{q}} \tilde{\alpha}_{m} e^{-\jmath\left(2 \pi f_{o} \frac{l_{m}}{c}+\beta\right)} p\left(l T_{c}-\tau_{k, q}\right) \mathrm{a}\left(\theta_{m}, f_{o}\right) \\
& =\sum_{q=1}^{Q} \mathbf{v}_{k, q} p\left(l T_{c}-\tau_{k, q}\right)
\end{aligned}
$$

where $f_{0}$ is the uplink carrier frequency, $p(t)$ is given by the convolution of the modulation pulse shape and the receiver filter response, and $\mathbf{a}\left(\theta_{m}, f_{0}\right)$ is the vector of receive antenna gains and phases in azimuth direction $\theta_{m n}$ at frequency $f_{o}$. When the mobile moves, the propagation delay changes this causing the taps $\mathbf{c}_{k l}$ to be time-varying (Doppler-fading). In the second expression, $\mathbf{v}_{k, q}$ is given by the superposition of the steering vectors belonging to cluster $q$ associated to user $k$, i.e.

$$
\mathbf{v}_{k, q}=\sum_{m \in \Omega_{c}} \tilde{\alpha}_{m} e^{-\jmath\left(2 \pi f_{o} \frac{l m_{m}}{c}+\beta\right)} \mathbf{a}\left(\theta_{m}, f_{0}\right) .
$$

For a number of scatterers, the vectors $\mathbf{v}_{k, q}$ can be regarded as zero-mean complex Gaussian-distributed wide-sense stationary random processes with a limiting multipath covariance matrix given by [4]:

$$
\mathbf{R}_{k, q}=E\left[\mathbf{v}_{k, q} \mathbf{v}_{k, q}^{H}\right]==\sum_{m \in \Omega_{c}}\left|\tilde{\alpha}_{m}\right|^{2} \mathbf{a}\left(\theta_{m}, f_{0}\right) \mathbf{a}^{H}\left(\theta_{m}, f_{0}\right)
$$

Assuming the number of scatterers within each cluster is infinite (Fig. 2), several power azimuthal distributions can be devised in order to match those commonly observed. Departing from the well-known approach that employs a truncated Gaussian Angle of Arrival (GAA) distribution [4], a Laplacian Angle of Arrival (LAA) pdf will be considered here. As shown in [5], the power azimuth distribution is well modelled with the Laplacian function in a variety of environments where the Gaussian distribution fails in reproducing the sharp peak at $0^{\circ}$. In practice, the distribution will be restricted to the range $\bar{\theta}_{m} \pm 2 \sigma$, where $\bar{\theta}_{m}$ is the mean DOA and $\sigma$ stands for the corresponding standard deviation (angular spread). Typical values for the latter parameter lie in the range $2^{\circ}-8^{\circ}$.

\subsection{Angular Characterisation of Standard Test Channels}

In order to obtain wideband channel models characterised both in the angular and delay domains, two approaches can be considered: adopting a scatterers' deployment (with resolvable delays) such that this matches both distributions or, alternatively, applying the narrowband models of the previous section to each tap in the delay profile to be generated. The latter strategy, which relies on the assumption that azimuth and delay dispersions are not coupled, will 


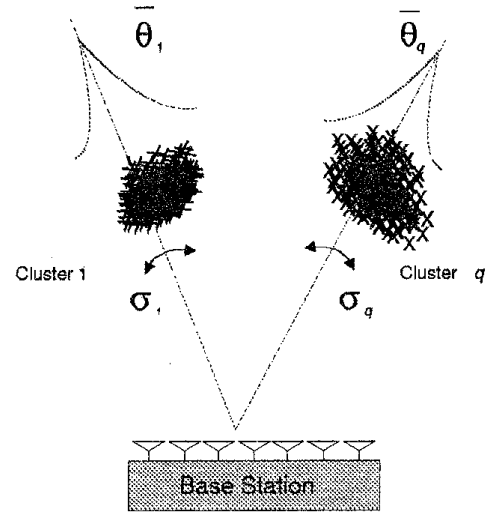

Figure 2. LAA propagation model.

be considered in the sequel. In particular, two tapped- delay lines proposed in [6] are taken into account (Fig. 3) The first one, Vehicular test Channel (Channel A), is more
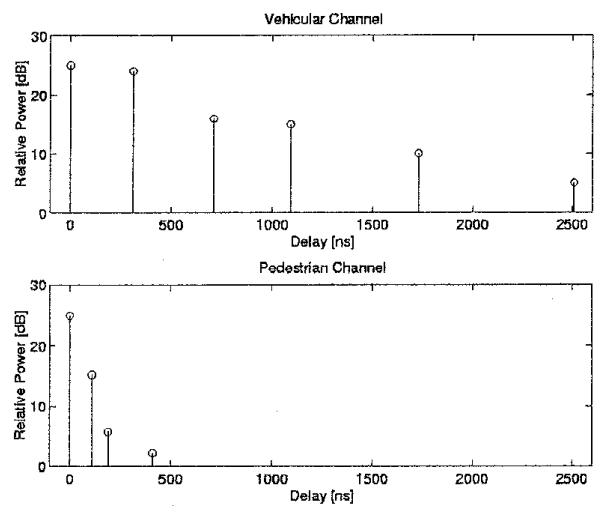

Figure 3. Tapped-delay lines for the pedestrian and vehicular channels.

appropriate to model situations where large cells and high transmitted power are used. The second model, Outdoor to Indoor and Pedestrian test channel (Channel A), models an environment characterised by smaller cells and low power base and mobile stations. Base stations with low antenna heights are located outdoors whereas pedestrian users are located both on streets and inside buildings. In all cases, a single-cluster LAA distribution has been assumed for the leading taps whereas an omnidirectional angular distribution has been adopted for the last (pedestrian model) or the last two taps (vehicular model). This way, reflections of distant scatterers are embedded in the channel model. Since only link-level simulations are presented in this paper, propagation models do not include slow-fading or path-loss effects. In accordance with $[7,8]$, these phenomena should be taken into account in system-level simulations.

\section{ALGORITHM DESCRIPTION}

Two array processing schemes are presented in this section. They represent a family of algorithms which jointly exploit or compensate the spatial and temporal characteristics of the propagation channel. Therefore they are only applicable to the uplink of a cellular communication system.

Both schemes belong to a class of algorithms in which a narrowband beamformer, $w$, is placed prior to the detection stage (see Figs. 4 and 5). The complex baseband components corresponding to each of the antenna elements are combined into a single complex signal, which is fed into a specific data detection device. For the design of the beamformer weights, the following expression is minimized:

$$
J(\mathrm{w}, \mathrm{c})=E\left[\left\|\mathrm{w}^{H} \mathrm{r}[n]-\mathrm{c}^{H} \mathrm{~s}[n]\right\|^{2}\right]
$$

where $\mathrm{c}$ is the $K^{r d} L \times 1$ vector constructed by stacking the physical channel response for the $K^{d}$ users of interest after spatial filtering (to be estimated along with the beamformer). On the other hand, $s[n]$ is a $K^{d} L M \times 1$ vector built from the last $L M$ chips in the training sequence for every user to be detected. Minimization is carried out with the restriction $\|\mathbf{c}\|=1$ so as to avoid the trivial solution. After some algebra, it can be obtained that the (multiuser) channel estimate, cmse, is given by the eigenvector associated to the smallest eigenvalue of the matrix

$$
\mathbf{F}=\mathbf{R}_{s s}-\mathbf{R}_{r s}^{H} \mathbf{R}_{r r}^{-1} \mathbf{R}_{r s}
$$

In the above expression, $\mathbf{R}_{s s}, \mathbf{R}_{r s}$ and $\mathbf{R}_{r r}$ stand for the autocorrelation and cross-correlation matrices for the transmitted and received data. Finally, the beamformer weights are computed from the recently obtained channel estimate:

$$
\mathbf{w}_{\text {mse }}=\mathbf{R}_{r r}^{-1} \mathbf{R}_{r s} \mathbf{c}_{\text {mse }}
$$

This common framework will now be particularized for the algorithms to be compared. For the FTS-JDR approach (Fig. 4), the number of users of interest, $K^{d}$, equals the number of intracell users. This way, the beamformer concentrates its efforts in exclusively cancelling out intercell interference whereas intracell users can be jointly detected following, for instance, a MMSE detection strategy [9].

In contrast, the second algorithm (FTS-MR, Fig. 5), aims to attenuate both intercell and the remaining intracell interferers in the beamforming stage. In consequence, $K^{d}=1$ now and, provided that a reduced number of users are to be detected, a MLSE strategy can be employed instead. No optimality claims can be made with respect to the performance of this scheme in any practical situation in which interferences are not completely cancelled. Nevertheless, the algorithm has shown good behaviour under realistic simulation tests and experimental data [10].

For each scheme, knowledge of the midamble training sequences as well as spreading sequences of every user to be detected is required.

\section{SIMULATION RESULTS}

For this section, we propose a scenario consisting in 8 asynchronous intracell users plus $K^{u}=1 \ldots 5$ intercell interferers impinging on a uniform linear array with $P=8$ elements $(\lambda / 2$-spaced $)$. A uniform distribution within the range $-60^{\circ}, \ldots, 60^{\circ}$ was adopted for the mean DOAs of 


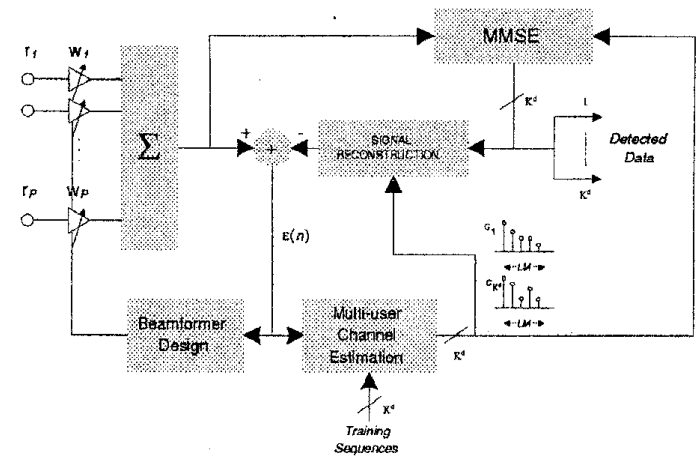

Figure 4. Filtered Training Sequence Joint Detection Receiver.

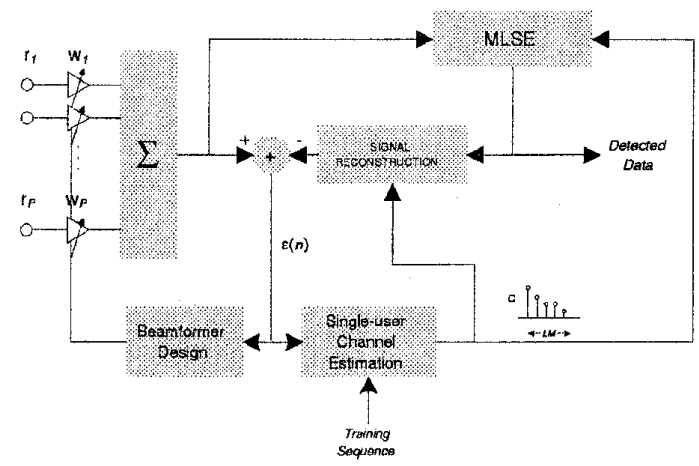

Figure 5. Filtered Training Sequence Multisensor Receiver.

both desired and interfering users. That is, a $120^{\circ}$ sectorization was assumed. Regarding angular spread, two cases were considered: low azimuth spread equal to $2^{\circ}$ for the Vehicular test channel and, moderate azimuth spread equal. to $8^{\circ}$ for the Pedestrian test channel. Additionally, two interfering scenarios were simulated for each channel model depending on the existence or not of a dominating intercell interferer. For the first case, a single intercell interferer was simulated whereas five interferers were generated in the second case. In all situations, the SNR for the AWGN noise was $20 \mathrm{~dB}$. The mobiles' speed was $3 \mathrm{~km} / \mathrm{h}$ for the pedestrian channels and $80 \mathrm{~km} / \mathrm{h}$ for the vehicular environment.

For spreading purposes, a number of $M=16$ chips per: symbol was chosen since this allows the use of orthogonal codes. Each burst contains two blocks of encoded data with a training sequence in-between. For all the active users, training sequences can be obtained as time-shifted versions of a single periodic pseudo-noise generating stream [3]. The spreading modulation is linearized GMSK whereas the data modulation is QPSK $(T=7.38 \mu \mathrm{s})$.

First of all, we present two examples of beamformers designer with the criterion expressed in equations 5-7 (Fig. 6 ). The plot on the top (FTS-JDR algorithm) reflects how intracell users are accepted whereas the intercell interferer is cancelled out. In contrast, when using the FTS-MR approach (bottom), all interferers are attenuated as much as possible.

Performance curves are presented in Fig. 7 where the uncoded (raw) BER is depicted as a function of the instantaneous (in-burst) signal to intracell plus intercell interference ratio. Several conclusions can be drawn. First, comparative performance for the algorithms under study clearly depends on the instantaneous C/I ratio. Regardless of the test channel and the total number of interferers, the scheme with a JD block performs better for very low $\mathrm{C} / \mathrm{I}$ ratios. In practical situations, however, a raw BER in the range should be guaranteed. Therefore, the range of interest for C/I neatly lies ini the zone where the FTS-MR algorithm outperforms FTS-JDR. In other words, for a finite number of sensors $(P=8)$ better results can be obtained when all interferes are eliminated in the beamforming block instead of relying on a JD strategy (that, in principle, would be the optimal approach). Moreover, computational complexity, which depends on the dimension of matrix $\mathbf{F}\left(K^{d} L \times K^{d} L\right)$ is dramatically lower for the FTS-MR where $K^{d}=1$.

Finally, several comments on the algorithms performance vs. the test environment set. It can be appreciated that, as the number of intercell interferers increases, performance gets worse. Actually, when $K^{u}=5$ the total number of active users is 13 so that, by no means, they will be completely eliminated by a 8-sensor array. On the other hand, performance loss turning out when moving to the PEDx to VEHx channels is due, mainly, to the time-varying nature accentuated by the increase of Doppler spread.

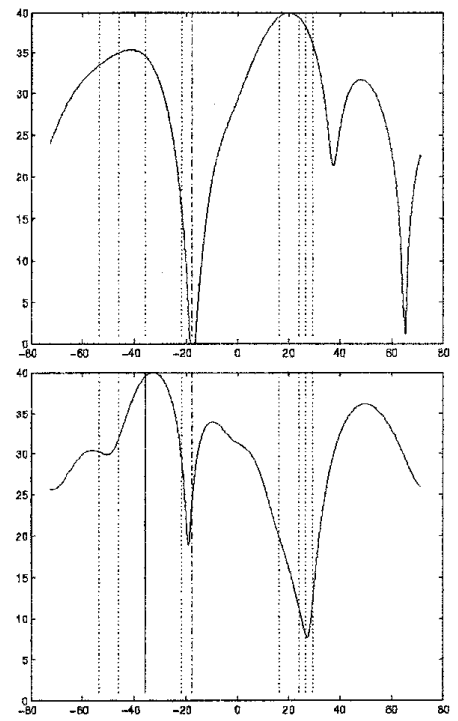

Figure 6. Beamformers' spatial response: FTS-JDR (top) and FTS-MR (bottom). Dotted lines: intracell users, dash-dot: intercell interferers, solid: desired intracell user.

\section{CONCLUSIONS}

In this paper, two array-based algorithms for the detection of DS-C/TDMA signals have been presented and compared in standard UMTS test scenarios. Simulation results indicate that for the adopted configuration, better results can be obtained by the FTS-MR algorithm where no joint detection mechanism is employed. Moreover, computational 

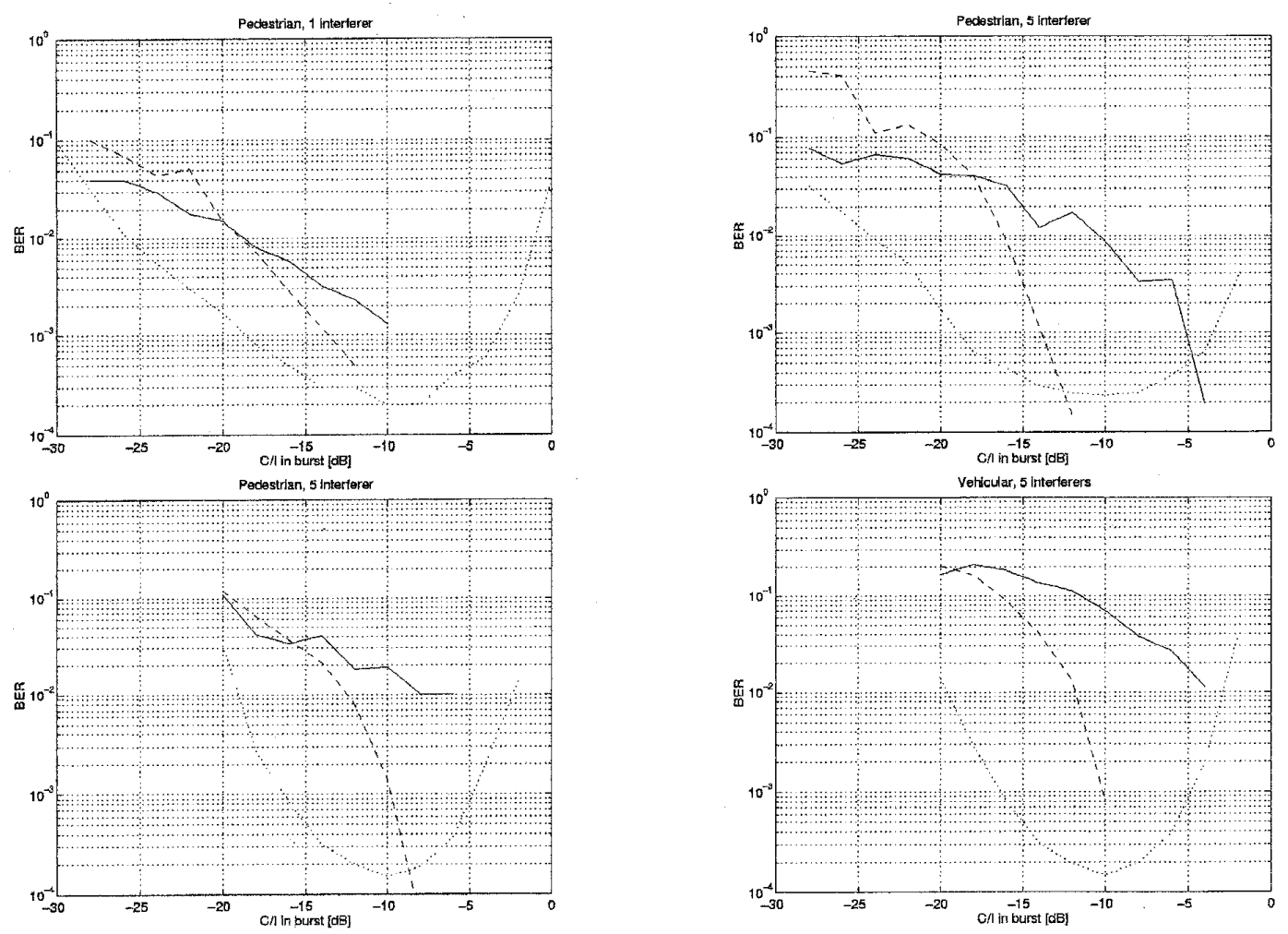

Figure 7. BER vs. C/I for different test environments: FTS-JDR algorithm (solid-line), FTS-MR algorithm (dashed line). The confidence ratio, defined as ten times the inverse of the number of symbols associated with each instantaneous $\mathrm{C} / \mathrm{I}$ value, is represented by the dotted line.

complexity is substantially lower for this approach than for the FTS-JDR scheme.

\section{REFERENCES}

[1] DTR/SMG-050101, "Objectives and overview of UMTS," tech. rep., ETSI, 1992.

[2] DTR/SMG-050103,

"System requirements for UMTS," tech. rep., ETSI, 1992.

[3] T. Ojanperä, P. Anderson, J. Arnbak, J. Castro, L. Girard, A. Klein, and J. Skold, "Future radio wideband multiple access system - FRAMES," R AC090/NOK/A10/DS/R/008/a1, ACTS 090, Nov. 1997.

[4] P. Mogensen, P. Zetterberg, H. Dam, P. Leth-Espesen, and F. Frederiksen, "TSUNAMI II: algorithms and antenna array recommendations (Part 1)," Tech. Rep. AC020/AUC/A1.2/DR/P/005/a1, ACTS 020, Aalborg University AUC, Oct. 1996.

[5] K. Pedersen, P. Mogensen, B. Fleury, F. Frederiksen, K. Olesen, and S. Larsen, "Analysis of time, azimuth and doppler dispersion in outodoor radio channels," in Proc. ACTS Mobile Telecommunications Summit, (Aalborg (Denmark)), pp. 599 604, October 1997.
[6] E. Nikula et al., "Future radio wideband multiple access system - FRAMES," Tech. Rep. AC090/NOK/PT0/DS/R/014/b1, ACTS 090, June 1996.

[7] S. Hamalainen, P. Slanina, M. Hartman, A. Lappetelainen, H. Holma, and O. Salonaho, "A novel interface between link and system level simulations," in Proc. ACTS Mobile Telecommunications Summit, (Aalborg (Denmark)), pp. 605-610, October 1997.

[8] J. Wigard and P. Mogensen, "A simple mapping from c/i to fer and ber for a gsm type of air-interface," in Proc. of PIMRC 96, (Taipei (Taiwan)), September 1996.

[9] P. Jung and J. Blanz, "Joint detection with coherent receiver antenna diversity in CDMA mobile radio systems," IEEE Trans. on Vehicular Technology, vol. 44, pp. 76-88, Feb. 1995.

[10] F. Pipon, P. Chevalier, P. Villa, and J. Monot, "Joint spatial and temporal equalization for channels with isi and cci: Theoretical and experimental results for a base station reception," in Proc. IEEE Signal Processing Workshop on Signal Processing Advances in Wireless Communications, (Paris (France)), pp. 309-312, April 1997. 